\title{
FRAMING CURVES IN EUCLIDEAN AND MINKOWSKI SPACE
}

\section{ROBERT J. LOW}

Communicated by Gregory L. Naber

Abstract. We give a unified picture of the Frenet-Serret equations in Euclidean space and their analogues in Minkowski space that provides further insight into how and why the Minkowski versions differ from the Euclidean.

\section{Introduction}

The Frenet apparatus for a curve in Euclidean space $\mathbb{E}^{3}$ whose curvature vanishes nowhere is a standard part of the undergraduate introduction to differential geometry, and a description can be found in any introductory text, such as that of Pressley [3]. The usual naming convention for this orthonormal triad is $\{T, N, B\}$, where $T$ is (proportional to) the tangent vector, $N$ is proportional to the derivative of $T$, and $B$ completes a right-handed orthonormal basis. These are related by the Frenet-Serret equations

$$
\left[\begin{array}{l}
\dot{T} \\
\dot{N} \\
\dot{B}
\end{array}\right]=\left[\begin{array}{ccc}
0 & \kappa & 0 \\
-\kappa & 0 & \tau \\
0 & -\tau & 0
\end{array}\right]\left[\begin{array}{l}
T \\
N \\
B
\end{array}\right]
$$

Analogous framings can be found in the three dimensional Minkowski space $\mathbb{M}^{1,2}$, with signature $(-,+,+)$ and inner product $\langle.,$.$\rangle for curves which are everywhere$ timelike, everywhere spacelike, or everywhere null. The equations describing these framings can be derived in a similar way once a cross product has been defined and are similar to those of the Frenet-Serret equations frame, but with some changes of sign arising from the indefinite nature of the metric, as in Lopez [2].

For example, for a timelike curve, one obtains

$$
\left[\begin{array}{c}
\dot{T} \\
\dot{N} \\
\dot{B}
\end{array}\right]=\left[\begin{array}{ccc}
0 & \kappa & 0 \\
\kappa & 0 & \tau \\
0 & -\tau & 0
\end{array}\right]\left[\begin{array}{l}
T \\
N \\
B
\end{array}\right]
$$

where the sign of $\kappa$ in the second row changes because of the indefinite inner product. For curves of other causal characters there is a different pattern of signs, which can be found by an explicit calculation similar to that in Pressley [3]. 
The purpose of this paper is to give a unified picture in terms of moving frames which provides additional insight into the pattern of signs which occurs in these equations.

\section{Frames Adapted to a Curve}

Let $C: I \rightarrow \mathbb{R}^{3}$ be a differential mapping with domain $I$, an interval in $\mathbb{R}$. Then to each $s \in I$, we associate $F(s)$, a matrix whose columns are a basis for $\mathbb{R}^{3}$, and which we regard as attached to the point $C(s)$. Denote the columns of $F$ by $e_{1}, e_{2}, e_{3}$.

It follows that $F \in \mathrm{GL}(3, \mathbb{R})$. More interestingly, we can consider the case where $F$ represents a particular class of basis, such as an orthonormal basis, and so $F$ belongs to a subgroup of $\mathrm{GL}(3, \mathbb{R})$. In the case of an orthonormal triad, this will be the rotation group, $\mathrm{SO}(3)$. Denote by $H$ the subgroup of matrices whose columns are the triads of interest.

We want to describe how this basis evolves along the curve, so fix some $s_{0} \in I$. We need to know $\dot{F}\left(s_{0}\right)$. If we denote $F\left(s_{0}\right)$ by $B$, then we can consider the curve of matrices $A(s)=B^{-1} F(s)$, so that $A\left(s_{0}\right)$ is the identity. Differentiating this, we see that $\dot{A}=B^{-1} \dot{F}$, so that $\dot{F}\left(s_{0}\right)=B \dot{A}\left(s_{0}\right)=F\left(s_{0}\right) \dot{A}\left(s_{0}\right)$, where $\dot{A}\left(s_{0}\right)$ is an element of the Lie algebra of $H$. Denoting $\dot{A}\left(s_{0}\right)$ by $\mathcal{A}$ we thus have

$$
\dot{e}_{i}=\sum_{j=1}^{3} e_{j} \mathcal{A}_{j i}
$$

which can therefore be re-written as

$$
\left[\begin{array}{c}
\dot{e}_{1} \\
\dot{e}_{2} \\
\dot{e}_{3}
\end{array}\right]=\Omega\left[\begin{array}{l}
e_{1} \\
e_{2} \\
e_{3}
\end{array}\right]
$$

where $\Omega=\mathcal{A}^{t}$.

We can now immediately see a major difference between the Euclidean and Minkowskian signatures. All orthonormal bases in the Euclidean case give rise to the same group of matrices, namely those whose transpose is their inverse. This is the reason for the skew-symmetric nature of the Frenet-Serret equations. However, in the case of Minkowski space, even when the basis contains one timelike and two spacelike unit vectors, the choice of order changes the signs in the metric, and consequently changes the signs in the Frenet-Serret equations. We also have to contend with the case where the basis may contain null vectors, and will not be orthonormal. 
In each case, however, the metric with respect to the chosen basis $\left\{e_{1}, e_{2}, e_{3}\right\}$ will be given by the matrix $\eta$, where $\eta_{i j}=\left\langle e_{i}, e_{j}\right\rangle$, the inner product of $e_{i}$ and $e_{j}$.

We will now see how the structure of the matrices $\Omega$ which correspond to elements of the Lie algebra determines the Frenet-Serret equations in each case of interest.

In each case, we will find form of the general evolution equation, and then restrict our attention to frames where $e_{1}$ is parallel to $\dot{C}$. Such frames are said to be adapted to the curve $C$, see Sternberg's notes on semi-Riemannian geometry [4] for an accessible treatment placing these ideas in a much more general context.

\section{The Frenet-Serret Equations in $\mathbb{E}^{3}$}

First, we see how the traditional Frenet-Serret equations arise in this context.

We look at frames which are orthonormal with respect to the standard Euclidean metric and adapted to the curve $C$, which is parameterized by arc-length. Then our frames give matrices in $\mathrm{SO}(3)$, and as is well-known [5], the Lie algebra consists of skew-symmetric matrices.

From this, since the transpose of a skew-symmetric matrix is skew-symmetric, we obtain evolution equations of the form

$$
\left[\begin{array}{c}
\dot{e}_{1} \\
\dot{e}_{2} \\
\dot{e}_{3}
\end{array}\right]=\left[\begin{array}{ccc}
0 & \Omega_{12} & \Omega_{13} \\
-\Omega_{12} & 0 & \Omega_{23} \\
-\Omega_{13} & -\Omega_{23} & 0
\end{array}\right]\left[\begin{array}{l}
e_{1} \\
e_{2} \\
e_{3}
\end{array}\right] .
$$

Making the further constraint that $e_{2}$ be proportional to $\dot{e}_{1}$ we find that $\Omega_{13}=0$, and so we obtain

$$
\left[\begin{array}{c}
\dot{e}_{1} \\
\dot{e}_{2} \\
\dot{e}_{3}
\end{array}\right]=\left[\begin{array}{ccc}
0 & \Omega_{12} & 0 \\
-\Omega_{12} & 0 & \Omega_{23} \\
0 & -\Omega_{23} & 0
\end{array}\right]\left[\begin{array}{l}
e_{1} \\
e_{2} \\
e_{3}
\end{array}\right]
$$

which we recognize as the familiar Frenet-Serret equations, with $\kappa=\Omega_{12}$ and $\tau=\Omega_{23}$.

\section{The Frenet-Serret Equations in $\mathbb{M}^{1,2}$}

Let us now consider the case of a curve in three-dimensional Minkowski space. It is possible to follow through the standard treatment in Euclidean space by introducing an appropriate notion of cross product, as in Yilmaz [7]. However, the purpose of this article to is provide an alternative derivation which uncovers the 
origin of the pattern of zeros and signs in the Frenet-Serret equations in a unified way.

A catalogue of the possible types of curve, categorized by the causal nature of the tangent and acceleration, and the corresponding Frenet-Serret equations, can be found in the doctoral thesis of Walrave [6] and I will follow his sign and notational conventions in order to make the comparison of results straightforward.

\subsection{Timelike Curves}

First, we consider the case where $\dot{C}$ is timelike, and $C$ is parameterized by proper time. It then follows that $e_{2}$ and $e_{3}$ are spacelike, and so the matrix $\eta$, describing the inner product of Minkowski space in this basis, is given by

$$
\eta=\left[\begin{array}{rrr}
-1 & 0 & 0 \\
0 & 1 & 0 \\
0 & 0 & 1
\end{array}\right]
$$

Thus our frame $F$ is required to satisfy the equation $F^{t} \eta F=\eta$.

To find the form of an element of the Lie algebra of this matrix group, we consider a curve of such matrices $A(s)$ such that $A(0)$ is the identity. Differentiating yields

$$
\dot{A}^{t}(0) \eta+\eta \dot{A}(0)=0
$$

and taking the general form of $\dot{A}(0)$ to be

$$
\left[\begin{array}{lll}
A_{11} & A_{12} & A_{13} \\
A_{21} & A_{22} & A_{23} \\
A_{31} & A_{32} & A_{33}
\end{array}\right]
$$

we obtain

$$
\left[\begin{array}{rrr}
A_{11}-A_{21} & -A_{31} \\
A_{12}-A_{22} & -A_{32} \\
A_{13} & -A_{23}-A_{33}
\end{array}\right]=\left[\begin{array}{rrr}
-A_{11} & -A_{12} & -A_{13} \\
A_{21} & A_{22} & A_{23} \\
A_{31} & A_{32} & A_{33}
\end{array}\right]
$$

so that

$$
A=\left[\begin{array}{ccc}
0 & A_{12} & A_{13} \\
A_{21} & 0 & A_{23} \\
A_{31} & -A_{32} & 0
\end{array}\right]
$$

which finally gives

$$
\Omega=\left[\begin{array}{ccc}
0 & \Omega_{12} & \Omega_{13} \\
\Omega_{21} & 0 & \Omega_{23} \\
\Omega_{31} & -\Omega_{23} & 0
\end{array}\right]
$$


We now make the further constraint that $e_{2}$ is proportional to $\dot{e}_{1}$, so we can set $\Omega_{13}=0$, and we obtain

$$
\left[\begin{array}{l}
\dot{e}_{1} \\
\dot{e}_{2} \\
\dot{e}_{3}
\end{array}\right]=\left[\begin{array}{ccc}
0 & \Omega_{12} & 0 \\
\Omega_{12} & 0 & \Omega_{23} \\
0 & -\Omega_{23} & 0
\end{array}\right]\left[\begin{array}{l}
e_{1} \\
e_{2} \\
e_{3}
\end{array}\right]
$$

which recovers the usual Frenet-Serret equations for a timelike curve as given in Walrave [6], when we make the identification $\Omega_{12}=\kappa_{1}$ and $\Omega_{23}=\kappa_{2}$.

\subsection{Spacelike Curves}

If $e_{1}$ is spacelike, then there are three possibilities: $\dot{e}_{1}$ may be timelike, spacelike, or null.

\subsubsection{Timelike Acceleration}

In the first case, we must replace $\eta$ by

$$
\left[\begin{array}{rrr}
1 & 0 & 0 \\
0 & -1 & 0 \\
0 & 0 & 1
\end{array}\right]
$$

and following through the above procedure we obtain

$$
\Omega=\left[\begin{array}{ccc}
0 & \Omega_{12} & \Omega_{13} \\
\Omega_{12} & 0 & \Omega_{13} \\
-\Omega_{13} & \Omega_{23} & 0
\end{array}\right]
$$

and again, restricting to the case where $e_{2}$ is parallel to $\dot{e}_{1}$, we have

$$
\Omega=\left[\begin{array}{ccc}
0 & \Omega_{12} & 0 \\
\Omega_{12} & 0 & \Omega_{23} \\
0 & \Omega_{23} & 0
\end{array}\right]
$$

which agrees with Walrave with $\Omega_{12}=\kappa_{1}$ and $\Omega_{23}=\kappa_{2}$.

\subsubsection{Spacelike Acceleration}

In the second case our metric takes the form

$$
\left[\begin{array}{rrr}
1 & 0 & 0 \\
0 & 1 & 0 \\
0 & 0 & -1
\end{array}\right]
$$


Carrying out the analogous calculation, we obtain

$$
\Omega=\left[\begin{array}{ccc}
0 & \Omega_{12} & \Omega_{13} \\
-\Omega_{12} & 0 & \Omega_{23} \\
\Omega_{13} & \Omega_{23} & 0
\end{array}\right] .
$$

Again requiring $e_{2}$ to be parallel to $\dot{e}_{1}$, we obtain

$$
\Omega=\left[\begin{array}{ccc}
0 & \Omega_{12} & 0 \\
-\Omega_{12} & 0 & \Omega_{23} \\
0 & \Omega_{23} & 0
\end{array}\right]
$$

and we identify $\Omega_{12}$ and $\Omega_{13}$ as $\kappa_{1}$ and $\kappa_{2}$ to recover Walrave's result.

\subsubsection{Null Acceleration}

In the third case, we have to consider a suitable replacement for an orthonormal triad which includes a null vector. The natural triad to use is of the form $\left(e, n_{1}, n_{2}\right)$, where the $n_{i}$ are null vectors such that $\left\langle n_{1}, n_{2}\right\rangle=1$, and $e$ is a unit spacelike vector orthogonal to each of $n_{1}$ and $n_{2}$, parallel to the tangent to our curve.

Finding the metric in this basis, we now replace $\eta$ by

$$
\left[\begin{array}{lll}
1 & 0 & 0 \\
0 & 0 & 1 \\
0 & 1 & 0
\end{array}\right]
$$

which yields a matrix of the form

$$
\Omega=\left[\begin{array}{ccc}
0 & \Omega_{12} & \Omega_{13} \\
\Omega_{13} & \Omega_{22} & 0 \\
\Omega_{12} & 0 & -\Omega_{22}
\end{array}\right]
$$

and it is noteworthy that since the metric is not represented by a diagonal matrix, the Lie algebra loses its semi-skew symmetric nature. Nevertheless, we can proceed just as before.

Denoting $\left(e, n_{1}, n_{2}\right)$ by $\left(e_{1}, e_{2}, e_{3}\right)$, this time, we define $e_{2}$ by $e_{2}=\dot{e}_{1}$, so that $\Omega_{13}=0$ and $\Omega_{12}=1$. Finally we identify $\Omega_{22}=\kappa_{2}$ to recover Walrave's result.

\subsection{Null Curve}

The final case is that of a null curve, so that $\dot{C}$ is null. As there is no notion of arclength here, we cannot normalize this curve by requiring that $\dot{C}$ have unit speed. 
Instead, we note that (as long as $C$ is not a null geodesic), $\ddot{C}$ must be spacelike, so we will choose the parameter to make $\ddot{C}$ of unit length.

Since we have at least one null basis vector, we cannot have an orthonormal triad. An obvious alternative is to choose a basis consisting of two null vectors $n_{1}$ and $n_{2}$ such that $\left\langle n_{1}, n_{2}\right\rangle=1$ and a spacelike unit vector orthogonal to each of them.

In this case, we will have $e_{1}=n_{1}$, the tangent to $C, e_{3}=n_{2}$, the null vector satisfying $\left\langle n_{1}, n_{2}\right\rangle=1$, and $e_{2}=e$, the unit spacelike vector orthogonal to both $n_{1}$ and $n_{2}$.

In this basis, the metric is given by

$$
\left[\begin{array}{lll}
0 & 0 & 1 \\
0 & 1 & 0 \\
1 & 0 & 0
\end{array}\right]
$$

and we obtain

$$
\Omega=\left[\begin{array}{ccc}
\Omega_{11} & \Omega_{12} & 0 \\
\Omega_{21} & 0 & -\Omega_{12} \\
0 & -\Omega_{12} & -\Omega_{11}
\end{array}\right]
$$

If we choose the parameter so that $\dot{n}_{1}$ is a unit vector, we can take $e_{2}=\dot{e}_{1}$, and we have $\Omega_{11}=0$, and $\Omega_{12}=1$. Hence we obtain

$$
\Omega=\left[\begin{array}{ccr}
0 & 1 & 0 \\
\Omega_{12} & 0 & -1 \\
0 & -\Omega_{12} & 0
\end{array}\right]
$$

and identifying $\Omega_{12}$ with $\kappa_{2}$ we obtain Walrave's final case.

We thus see that the pattern of signs and zeros in the Frenet-Serret equations for curves in Minkowski space is determined by the pattern of signs and zeros of the inner products of the type of frame. But this is an indirect dependance, via the Lie algebra of the matrix group keeping the matrix of inner products invariant, which allows the pattern to be different even in cases where the basis has one timelike and two spacelike vectors.

\section{Other Framings}

As Bishop [1] pointed out, there is more than one way to frame a curve. In the case he investigates in Euclidean space, the vector $e_{2}$ is chosen so that $\dot{e}_{2}$ and $\dot{e}_{3}$ are parallel to $e_{1}$. In our approach, this corresponds to insisting that $\Omega_{23}=0$ instead of requiring $\Omega_{13}=0$. Hence we immediately obtain Bishop's evolution equations with $\Omega_{12}=k_{1}, \Omega_{13}=k_{2}$. 
We can carry out the analogous procedure in Minkowski space with a timelike or spacelike curve. If the acceleration vector is spacelike, set in $\Omega_{23}=0$ yields

$$
\Omega=\left[\begin{array}{ccc}
0 & \Omega_{12} & \Omega_{13} \\
-\Omega_{12} & 0 & 0 \\
\Omega_{13} & 0 & 0
\end{array}\right]
$$

and again setting $\Omega_{12}=k_{1}, \Omega_{13}=k_{2}$ we recover the evolution equation for the Bishop frame as given in Yilmaz [7].

The case of a spacelike curve with timelike acceleration can be done in just the same way, yielding

$$
\Omega=\left[\begin{array}{ccc}
0 & \Omega_{12} & \Omega_{13} \\
\Omega_{12} & 0 & 0 \\
-\Omega_{13} & 0 & 0
\end{array}\right]
$$

Likewise, for a timelike curve, setting $\Omega_{23}=0$ gives

$$
\Omega=\left[\begin{array}{lll}
0 & \Omega_{12} & \Omega_{13} \\
\Omega_{12} & 0 & 0 \\
\Omega_{13} & 0 & 0
\end{array}\right] .
$$

Finally, we see immediately that for a null curve, we can only set $\dot{e}_{2}$ and $\dot{e}_{3}$ parallel to $e_{1}$ in the special case where the curve is a null geodesic and the frame is constant (so the curvatures all vanish).

\section{Conclusion}

We have seen a unified approach to finding the evolution equations for frames in Euclidean and Minkowski space. This shows clearly how the choice of a Frenet frame arises as a special case, and also allows the development of the Frenet-Serret equations in all the standard cases in Minkowski space, without the need to develop a cross product. In the process, it elucidates how the choice of class of basis affects the Frenet-Serret equations. The approach works equally well for obtaining the evolution equations for a Bishop frame, and for seeing that no such frame exists for a (non-geodesic) null curve. 


\section{References}

[1] Bishop R., There is More than One Way to Frame a Curve, Am. Math. Monthly 82 (1975) 246-251.

[2] Lopez R., Differential Geometry of Curves and Surfaces in LorentzMinkowski Space, Preprint arxiv:0810.3351v1 [math.DG] (2008).

[3] Pressley A., Elementary Differential Geometry, Springer, London 2001.

[4] Sternberg S., Semi-Riemann Geometry and General Relaivity, freely available at http://www. math. harvard/ shlomo/docs/semi_riemannian_geometry.pdf 2002.

[5] Tapp K., Matrix Groups for Undergraduates, AMS, Providence, 2005.

[6] Walrave J., Curves and Surfaces in Minkowski Space, PhD thesis, KU Leuven, Faculty of Science Leuven 2005.

[7] Yilmaz S., Bishop Spherical Images of a Spacelike Curve in Minkowski 3Space, International Journal of the Physical Sciences 56 (2010) 898-905.

Robert J. Low

Department of Mathematics

and Control Engineering

Coventry University

Priory Street

Coventry CV1 5FB

UK

E-mail address: mtx014@coventry.ac.uk 